\title{
Das Representações Numéricas Inatas à Matemática Culturalmente Construída
}

\author{
Paulo Estêvão Andrade ${ }^{1}$ \\ Departamento de Estudos Pedagógicos do Ensino Fundamental do Colégio Criativo, \\ Marília, SP, Brasil \\ Paulo Sérgio Teixeira do Prado \\ Departamento de Psicologia da Educação da Universidade Estadual Paulista \\ Júlio de Mesquita Filho, Marília, SP, Brasil \\ João dos Santos Carmo \\ Departamento de Psicologia da Universidade Federal de São Carlos, São Carlos, SP, Brasil \\ Instituto Nacional de Ciência e Tecnologia sobre Comportamento, Cognição e Ensino, \\ São Carlos, SP, Brasil
}

\section{Resumo}

Dados oriundos de investigações recentes em psicologia cognitiva, neuropsicologia e neurociência cognitiva tem permitido um crescente consenso de que as representações numéricas nos adultos e o pensamento matemático culturalmente construído dependem da interação entre o chamado "senso numérico", observado em bebês humanos e em outras espécies animais, e a linguagem. Na pesquisa com bebês, a técnica do "olhar preferencial" tem permitido a descoberta de habilidades sofisticadas em idades precoces, entre elas as de soma e subtração. A neuropsicologia apresenta casos de lesões em áreas do cérebro que causam prejuízos a habilidades específicas, com preservação de outras. E a neurociência cognitiva, por meio de modernos recursos de neuroimagem, tem permitido a localização precisa de áreas cerebrais responsáveis pelo processamento numérico. Todas essas contribuições lançam luzes sobre a natureza da cognição numérica subjacente à matemática. São apresentados e discutidos dados produzidos por pesquisas nas referidas áreas, bem como suas implicações para as teorias clássicas do desenvolvimento cognitivo e do conceito de número, em particular. Discute-se também a visão decorrente sobre a relação entre biologia e cultura, que passam a ser vistas não mais de maneira dicotômica e sim como continuidade uma da outra, bem como implicações pedagógicas e sobre a formação de educadores.

Palavras-chave: Senso numérico, representação numérica, pensamento matemático, modelos de cognição numérica.

\section{From Innate Numerical Representations to Culturally Constructed Mathematics}

\footnotetext{
Abstract

Data from recent research in cognitive psychology, neuropsychology and cognitive neuroscience has allowed a growing consensus that the numerical representations in adults and the culturally constructed

Endereço para correspondência: Departamento de Estudos Pedagógicos do Ensino Fundamental, Colégio Criativo, Rua das Safiras, 45, Jardim Maria Isabel, Marília, SP, Brasil 17516-023. Fone: (16) 98114-5194. E-mail: paulo_sustain@yahoo.com

Financiamento: Instituto Nacional de Ciência e Tecnologia sobre Comportamento, Cognição e Ensino, Apoiado pelo Ministério da Ciência, Tecnologia e Inovação (MCT) no âmbito do Edital 15/2008, com auxílio do Conselho Nacional de Desenvolvimento Científico e Tecnológico (CNPq, \#573972/2008-7) e da Fundação de Amparo à Pesquisa do Estado de São Paulo (FAPESP, \#2008/57705-8).
} 
mathematical thinking depend on the interaction between the so-called "number sense", observed in human babies and in other animal species, and the language. In research with babies, the technique of "preferential looking" has allowed the discovery of sophisticated skills in early ages, among them those of addiction and subtraction. The neuropsychology presents cases of lesions in areas of the brain that cause damage to specific skills, with preservation of others. And cognitive neuroscience, by means of neuroimaging features modern, has allowed the precise location of brain areas responsible for numeric processing. All these contributions shed light on the nature of numerical cognition underlying mathematics. Data produced by those areas are presented and discussed research, as well as its implications for the classical theory of cognitive development and the concept of number in particular. It discusses also the vision arising on the relationship between biology and culture, which will be seen no more as dichotomous way but as a continuation of each other, as well as pedagogical implications and on the training of educators.

Keywords: Number sense, numeric representation, mathematical thought, numerical cognition models.

\section{Desde las Representaciones Numéricas Innatas hacia la Matemática Culturalmente Construida}

\section{Resumen}

Datos de recientes investigaciones en psicología cognitiva, la neuropsicología y la neurociencia cognitiva han permitido un creciente consenso de que las representaciones numéricas en adultos y el pensamiento matemático culturalmente construido dependen de la interacción entre el llamado "sentido numérico", observados en bebés humanos y otras especies animales y la lengua. En la investigación con los bebés, la técnica de "mirada preferencial" ha permitido el descubrimiento de habilidades sofisticadas en edades tempranas, entre ellos los de suma y resta. La neuropsicología presenta casos de lesiones en áreas del cerebro que causan daño a las habilidades específicas, con la preservación de las demás. Y la neurociencia cognitiva, mediante modernos recursos de neuroimagen, ha permitido la ubicación precisa de las áreas del cerebro responsables por el procesamiento numérico. Todas estas contribuciones arrojan luz sobre la naturaleza de la cognición numérica subyacente a la matemática. Son presentados y discutidos datos de investigación producidos en esas áreas, así como sus implicaciones para la teoría clásica de desarrollo cognitivo y el concepto de número en particular. Se analiza también la visión que surge en la relación entre biología y cultura, que será vista no más como dicotómica sino como una continuación de ambas, así como implicaciones pedagógicas y respecto la formación de los educadores.

Palabras clave: Sentido numérico, representación numérica, pensamiento matemático, modelos de cognición numérica.

Há um crescente consenso de que as representações numéricas nos adultos e o pensamento matemático culturalmente construído dependem dainteraçãoentreum "sensonumérico"(Dehaene, 1997) presente em bebês desde tenra idade (Dehaene, Dehaene-Lambertz, \& Cohen, 1998; para uma revisão em português veja Andrade, 2006a, 2006b), e a linguagem (Andrade, 2006a; Carey, 2004; Gelman \& Butterworth, 2005; Spelke, 2003; Wynn, 1992a). O senso numérico é visto como constituído de dois mecanismos não verbais de percepção genuinamente numérica: um sistema exato para numerosidades pequenas (ou subitizáveis ${ }^{2}$ ) até quatro elementos, e um sistema aproximado para numerosidades maiores (Carey, 2004; Feigenson, Dehaene, \&

2 O termo "subitização" é derivado de "súbito" (repentino) e tem sido usado como referência à capacidade que temos para reconhecer e discriminar, em frações de segundo, conjuntos de até três ou quatro elementos sem o emprego da contagem. 
Spelke, 2004). O presente artigo apresenta um levantamento sobre essas concepções, baseado em investigações empíricas recentes sobre a natureza dos sistemas cognitivos subjacentes ao comportamento numérico.

\section{Paradigmas Teórico-Empíricos da Psicologia e Neurociência Cognitivas}

Piaget declarou que o primeiro ano de vida ainda era um abismo de mistérios para o psicólogo e que a observação do bebê em ação seria uma janela para descobrirmos o que se passa em sua mente e tudo o que há sobre psicologia (Lima, 1999). A moderna psicologia cognitiva vem trabalhando há décadas para descobrir, pelo menos em parte, o que acontece na mente dos bebês por meio do estudo detalhado e controlado do seu comportamento.

Desde meados dos anos 1960 se sabe da existência do "reflexo de orientação", presente nos bebês e, portanto, considerado inato nos humanos, de prestar mais atenção a estímulos novos do que aos familiares (Fantz, 1964). Se há esta preferência pela novidade, conclui-se que eles são capazes de perceber diferenças entre o novo e o velho. A técnica do olhar preferencial, baseada no reflexo de orientação, é conhecida como "habituação". Seu emprego consiste de mostrar-se uma figura ou objeto ao bebê repetidas vezes, até que se observe uma diminuição sensível no seu tempo de olhar, o que é tomado como indicativo de habituação, isto é, familiarização com o estímulo. Tendo a criança se habituado com um determinado estímulo, mostra-se um novo, no qual diferenças específicas serão manipuladas, tais como forma, cor, quantidade, etc. Se o bebê olhar significativamente por mais tempo para o estímulo novo, isso sugere que houve "desabituação": discriminação das diferenças entre o primeiro e o segundo estímulos. Esse paradigma tem permitido aos pesquisadores avaliar se o bebê é ou não capaz de discriminar atributos diversos, trazendo novas luzes sobre suas capacidades inatas. Graças a esse método e a outras novas técnicas de pesquisa, as três últimas décadas têm testemunhado um acúmulo de evidências notáveis de capacidades muito precoces, até mesmo em recém-nascidos. Elas sugerem, com muita eloquência, que o bebê humano possui uma percepção muito precoce do comportamento dos objetos no espaço e do próprio espaço (Spelke, 2003), bem como dos sons linguísticos (Kuhl, 2004), dos padrões musicais (Andrade, 2004; para uma revisão veja Andrade 2006a, 2006b; Andrade \& Prado, 2003; Prado, 2010) e da numerosidade (Hauser \& Spelke, 2004; Wynn, 1992a), sobre a qual nos concentraremos aqui.

\section{Pesquisa com Bebês: Estudos Clássicos}

Em 1992, K. Wynn publicou um dos mais importantes experimentos sobre numerosidade em bebês. Ela tomou 32 crianças de cinco meses de idade, em média, subdividindo-as em dois grupos iguais. Um deles, denominado " $1+1$ ", foi exposto a uma situação em que, inicialmente, apresentava-se um palco de fantoches vazio. Em seguida, por uma abertura lateral, uma mão com luva de um adulto introduzia um boneco. $\mathrm{Na}$ sequência, um anteparo era erguido à frente do palco, cobrindo a cena e outro boneco era visivelmente introduzido pela abertura lateral. Por um fundo falso, ainda com o anteparo levantado, um auxiliar de pesquisa podia remover um boneco sem que esta operação pudesse ser vista. Finalmente, o anteparo frontal era baixado, revelando dois bonecos - resultado correto -, ou um boneco - resultado incorreto. $\mathrm{O}$ outro grupo, denominado " 2 - 1", foi exposto a uma operação de subtração. A cena inicial apresentava a introdução de dois bonecos no palco. Ao ser levantado o anteparo, um era retirado de modo visível para os bebês pela abertura lateral. Quando o anteparo era, finalmente, baixado, o resultado podia ser o esperado: um boneco; ou inesperado: dois bonecos. Antecedendo essas operações, todos os bebês foram expostos a um e dois bonecos, mensurando-se seu tempo de olhar, o que serviu como medida de linha de linha de base para comparações ulteriores. Cada operação de adição ou subtração foi exibida seis vezes para cada participante dos dois grupos, alternando- 
-se o número de itens resultante entre um e dois. Ou seja, os resultados foram alternados entre correto e impossível, três vezes cada. Sendo a variável dependente o tempo de olhar, os dados foram comparados mediante o Teste $t$, revelando que os bebês olharam mais longamente para os resultados incorretos do que para os corretos, sendo essa diferença estatisticamente significante ao longo de todos os blocos de testes. Com base nesse resultado, a autora concluiu que a explicação mais plausível para ele é que os bebês podem efetuar operações aritméticas simples e que tal habilidade é inata, fornecendo a base para o desenvolvimento do conhecimento aritmético ulterior. Alguns pesquisadores levantaram a possibilidade de que em vez de estarem discriminando os arranjos com base na numerosidade, os bebês de Wynn (1992a) e de estudos semelhantes, poderiam estar esboçando uma preferência por estímulos com os quais já estivessem familiarizados e/ou pelos arranjos simplesmente com mais objetos (Cohen \& Marks, 2002).

No primeiro de três experimentos, replicando, com modificações, o procedimento de Wynn (1992a), Cohen e Marks (2002) expuseram 80 bebês de cinco meses a uma série de dois tipos de operações: " $1+1$ " e " $2-1$ ". Diferentemente do estudo original, em que houve alternância de dois resultados para cada uma daquelas operações: correto ou incorreto, os bebês de Cohen e Marks viam quatro, isto é, $0,1,2$ ou 3 objetos, perfazendo um total de oito pares de eventos numéricos e, portanto, seis incorretos e apenas dois corretos. Apesar dessas diferenças metodológicas, os resultados foram semelhantes aos obtidos por Wynn - maior tempo de olhar para o resultado incorreto tanto no grupo de adição $(1+1)$ quanto no de subtração $(2-1)$, no primeiro bloco de tentativas de teste. Entretanto, os participantes não mostraram preferência pelo resultado incorreto 3 em todos os blocos. Em suma, ao longo de todo o primeiro experimento, os bebês de Cohen e Marks olharam por mais tempo não para todos os resultados incorretos, mas preferiram os resultados de exibição idêntica à exibição inicial dos estímulos, que antecedeu a fase de testes.

Portanto, Cohen e Marks (2002) argumentaram que o maior tempo de olhar para os re- sultados incorretos no experimento de Wynn (1992a) poderia ser explicado pela preferência pela familiaridade com o padrão de estímulos da cena inicial. Num terceiro experimento, Cohen e Marks (2002) mostraram a 16 bebês, também de cinco meses, eventos de "mudança de número", mas sem qualquer operação aritmética, nos quais os bebês viam tanto 1 quanto 2 objetos serem escondidos por uma tela, cuja remoção, alguns segundos depois, podia revelar 0 , ou 1, 2 ou 3 itens. Aqui, os bebês novamente olharam por mais tempo para o resultado possível, no qual o número revelado era o mesmo que o da exibição inicial, do que para o resultado impossível.

No entanto, a hipótese da familiaridade levantada com base nesses dois experimentos de Cohen e Marks (2002) não explica porque os bebês de Wynn (1992a) também olharam por mais tempo para o resultado incorreto (3) após a operação " 1 + 1", além do resultado incorreto (1). Num segundo experimento, com 16 participantes com as mesmas características dos anteriormente descritos, os autores tentaram mostrar que a preferência por 3 após a operação " $1+1$ ", poderia ser explicada pela preferência por arranjos maiores e apresentaram diferentes arranjos de 0 , 1, 2 ou 3 itens, sem qualquer operação aritmética prévia, para os bebês simplesmente olharem para eles. Entretanto, no primeiro bloco os bebês olharam por mais tempo para 2 do que para 3 itens e a tendência de olharem por mais tempo os arranjos maiores começou a ocorrer somente no segundo bloco de tentativas. Mas, ainda assim, os dados desse segundo experimento foram tomados como evidência de que na adição " $1+$ 1", do estudo de Wynn (1992a), os bebês olharam por mais tempo para o resultado incorreto (3) do que no resultado correto, devido a uma preferência para números maiores e não porque tinham gerado uma expectativa de que o resultado seria 2 .

De fato, a hipótese da preferência por quantidades maiores cai por terra se levarmos em conta, primeiramente, que no próprio experimento 1 de Cohen e Marks (2002), os bebês não olharam significativamente por mais tempo para o par incorreto " $1+1=3$ " do que para o par correto " $1+$ $1=2$ " e, em segundo lugar, que a preferência por 
números maiores no segundo não emergiu antes de suas quatro primeiras tentativas.

Em suma, além de os resultados de Cohen e Marks (2002) não serem satisfatoriamente consistentes com sua hipótese de preferência pela familiaridade e pelos arranjos mais numerosos como explicação para os resultados de Wynn (1992a), eles também não foram suficientes para demostrar que as operações de adição e subtração não fazem diferença no paradigma padrão, em que há somente dois resultados ao invés de quatro (Carey, 2002). Em outras palavras, além de Cohen e Marks (2002) não terem produzido evidências consistentes com sua hipótese dual, eles não replicaram os resultados originais porque, como argumentaria posteriormente a própria Wynn (2002), o método utilizado por eles desvia-se do paradigma padrão de "violação de expectativa", no qual são equilibrados os resultados corretos e incorretos. Nas variações que introduziram, além do excesso de resultados diferentes - quatro, em contraste com os dois do estudo de Wynn (1992a) - houve também um grande desequilíbrio entre eventos impossíveis ( $75 \%$ das tentativas) e possíveis, em contraste com os $50 \%$ de eventos possíveis e $50 \%$ de impossíveis, do experimento de Wynn (1992a).

Essas variações podem ter significado a imposição de uma sobrecarga de informações, com um excesso de resultados diferentes e, consequentemente, de muitas possibilidades de escolhas, induzindo a atenção dos bebês para características perceptivas superficiais em detrimento das diferenças conceituais, cuja detecção requer processos inferenciais, os quais podem ser os primeiros a sofrerem prejuízos sob condições de sobrecarga de informação (Carey, 2002; Wynn, 2002).

Além do excesso de informação na exibição de quatro resultados diferentes, o delineamento experimental de Cohen e Marks (2002) também sofre do desequilíbrio entre uma maioria de $75 \%$ de eventos impossíveis, contra uma minoria de $25 \%$ de eventos possíveis, desequilíbrio este que, junto com o excesso de repetições e escolhas, pode levar os bebês a aprenderem rapidamente a "esperar o inesperado", ou que qualquer resultado é "possível" neste contexto experimental.
Ademais, esta hipótese não leva em conta que a descobertas de Wynn (1992a) geraram muitas replicações e extensões importantes, bem como novos paradigmas experimentais cujos resultados descartam definitivamente a hipótese opcional da preferência pela familiaridade (Andrade, 2006b).

\section{Evidências Robustas do Senso Numérico em Bebês e sua Natureza Supramodal ${ }^{3}$}

Há abundantes evidências oriundas de outros paradigmas experimentais para a permanência da representação de objetos escondidos e seus atributos numéricos em bebês pré-verbais, incluindo contextos empíricos que controlam eventuais efeitos de preferência pela familiaridade sensório-perceptiva.

Por exemplo, Simon, Hespos, e Rochat (1995) mostraram que os bebês responderam às transformações numéricas de objetos na tarefa de Wynn (1992a), mesmo quando as características desses objetos mudavam atrás da cortina, como, por exemplo, a substituição de um boneco por outro. Isto mostra que os bebês se basearam nas diferenças numéricas dos arranjos e não em diferenças sensoriais superficiais do objeto, como forma, cor, etc.

Koechlin, Dehaene e Mehler (1997) replicaram os resultados de Wynn (1992a), mostrando que os bebês respondiam ao número mesmo quando os objetos ocluídos se moviam sobre uma plataforma giratória, tornando sua localização variável e imprevisível, indicando, assim, que os bebês responderam às diferenças numéricas e não à localização dos objetos e, consequentemente, não à familiaridade sensório-perceptiva.

Aguiar e Baillargeon (1999) realizaram um estudo com palcos separados, no qual as preferências por familiaridade foram totalmente

3 O neologismo "supramodal" é empregado na língua inglesa, mais particularmente por integrantes da comunidade científica, como referência àquilo que transcende qualquer modalidade sensorial específica (visual, auditiva, olfativa, etc.). 
controladas e ainda assim bebês de apenas 2,5 meses distinguiram entre eventos com um e dois objetos e olharam mais tempo para resultados incorretos e não para os arranjos perceptivos familiares. Da mesma forma, bebês de 6 a 8 meses de idade distinguem a numerosidade não somente de objetos, mas também de eventos ou ações, como a mudança no número de pulos de um fantoche (Wynn, 1996). Portanto, a discriminação dos atributos numéricos de eventos ou ações é de difícil conciliação com a hipótese de preferência pela familiaridade de Cohen e Marks (2002).

Os bebês também são sensíveis à numerosidade contida em informações auditivas e, ainda, são capazes de integrar informações numéricas nas modalidades visual e auditiva. Starkey, Spelke, e Gelman (1990) mostraram a bebês diapositivos com 2 ou 3 objetos que produzem 2 ou 3 sons. Ao ouvirem duas batidas os bebês olham por mais tempo ao slide com 2 objetos e, da mesma forma, ao ouvirem 3 batidas eles olham por mais tempo para os slides contendo 3 objetos. Conclui-se que bebês de 6 a 8 meses podem parear objetos e sons e relacionarem a numerosidade contida em informações de modalidades sensórias diferentes.

Recentemente, Kobayashi, Hiraki, Mugitani, e Hasegawa (2004) replicaram as operações de Wynn (1992a) envolvendo objetos acústicos e visuais. Bebês de cinco meses entendem que a adição de 1 objeto visual +1 tom é igual a 2 objetos (ou eventos) e, da mesma forma, esperam que 1 objeto visual +2 tons é igual a 3 objetos, bem como 2 objetos visuais +1 tom também é igual a 3 objetos. A capacidade de reconhecer operações aritméticas básicas com estímulos de diferentes modalidades sensoriais indica fortemente que esta capacidade numérica não é o reflexo de uma simples tendência de preferir arranjos sensoriais e perceptivos gerais familiares ou mais complexos. Além disso, também indica que a percepção numérica é de natureza supramodal.

Huntley-Fenner, Carey e Solimando (2002), usando o mesmo paradigma de Wynn (1992a) mostraram a bebês de 8 meses de idade even- tos de adição " $1+1=2$ " (possível ou correto) ou 1 (impossível ou incorreto) empregando três diferentes tipos de material: dois objetos coesos, sendo um rígido e outro flexível, e um objeto não coeso, isto é, uma porção de areia. Os dois objetos coesos foram preparados para imitarem os mesmos atributos sensório-perceptivos superficiais de uma porção de areia. Isto é, foram cobertos com areia (técnica de colagem) de modo a apresentarem a mesma aparência, forma e volume de uma porção/pilha de areia, mas possuíam uma corda fixada em sua extremidade superior para poderem ser suspensos e baixados revelando sua coesão e solidez.

Neste estudo de Huntley-Fenner e colegas (2002) os bebês olharam consistentemente por mais tempo para os resultados impossíveis nas adições de objetos coesos, mas não para os eventos impossíveis para as porções de areia despejadas no palco. Em todas as tentativas deste estudo os bebês primeiramente sempre viram uma única entidade no palco, isto é, um objeto coeso ou uma porção de areia despejada, antes que esta fosse coberta por uma tela, de modo que após a adição de mais uma entidade idêntica os resultados impossíveis ou incorretos corresponderiam aos resultados idênticos ao estímulo inicial ou familiares, de modo que, de acordo com a hipótese da familiaridade de Cohen e Marks (2002), os bebês deveriam olhar por mais tempo aos resultados incorretos em ambas as séries com objetos coesos e porções de areia. $\mathrm{O}$ fato de os bebês terem olhado por mais tempo para os resultados incorretos somente nas séries com objetos coesos, sugere fortemente que este comportamento não reflete a preferência pela familiaridade, como proposto por Cohen e Marks (2002), mas sim por outra coisa, provavelmente a numerosidade.

Um fato importante do estudo de Huntley-Fenner et al. (2002) é que os bebês falham em construir representações de indivíduos não coesos como porções de areia, ou seja, em formar a representação de uma porção de areia como um indivíduo particular que pode ser rastreado no tempo e no espaço, mas são bem sucedidos com objetos coesos. 
De fato a coesão é usada por todas as culturas para distinguir não somente entre objetos individuais e coleções de objetos, mas também para distinguir objetos de substâncias não coesas ou não sólidas, como areia e água, respectivamente, distinção que se reflete nos sistemas nominais de muitas línguas do mundo, que marcam a distinção entre entidades que podem ser diretamente contadas de entidades não contáveis (Huntley-Fenner et al., 2002). Por exemplo, nós podemos dizer três cães porque o nome "cão" é um "nome contável" (count name), isto é, que se refere a um objeto individual e coeso e, portanto, contável, mas não podemos dizer "uma areia" ou "três areias" ou ainda "uma água" ou "três águas", a não ser que estes "nomes de massa" (mass names) sejam acompanhados de palavras de medida ou classificadora, tais como "dois montes de areia" ou "dois copos d'água" (Carey, 1997; Huntley-Fenner et al., 2002).

Realmente, os adultos parecem construir naturalmente o conceito de que materiais como areia e água não são indivíduos ou entidades não-individuadas. Carey (1997) nota que todas as línguas marcam a distinção gramatical entre objetos "individuáveis" e objetos não individuáveis, apesar da variação entre as línguas quanto às entidades contempladas por essa distinção gramatical. Por exemplo, há línguas que individuam somente pessoas, línguas que individuam também animais e objetos inanimados e línguas, como o português e o inglês, que incluem na sua individuação quaisquer estruturas complexas que possam ser rastreadas no tempo e no espaço como um todo coeso e coerente, bem como entidades abstratas (como um "cochilo" ou "uma opinião"; Carey, 1997). Portanto, a pesquisa sobre a permanência do objeto em bebês ainda muito novos e suas respectivas representações, bem como o rastreamento de objetos, tanto em bebês quanto em adultos, são abordagens cujos rótulos psicológicos são pertinentes e apropriados do ponto de vista comportamental, de modo que objetos coesos possuem um status privilegiado em relação aos objetos não coesos em nosso sistema neurocomputacional, pois estabelecem representações de indivíduos e os rastreiam no tempo e no espaço.

\section{O Paradigma da Busca Manual por Objetos}

Evidências muito convincentes sobre a natureza genuinamente numérica das respostas comportamentais dos bebês de Wynn (1992a) também foram produzidas em estudos posteriores com um novo paradigma não baseado na habituação, mas sim na busca manual por objetos.

À mesma época de Wynn (1992a), Starkey (1992) desenvolveu um método no qual crianças de 1 a 4 anos viram bolas de tênis sendo colocadas em uma caixa opaca e, depois, viram os experimentadores acrescentarem ou retirarem de uma a três bolas da caixa. Ao serem instadas a retirarem as bolas após as operações de adição ou subtração as crianças de um ano e meio a dois anos procuravam pelo número correto de bolas até a quantidade de 4, demonstrando uma compreensão dessas operações numéricas ainda em fase pré-verbal.

Este método de busca manual foi mais recentemente adaptado para crianças de 8 a 12 meses (Feigenson \& Carey, 2003; Van de Walle, Carey, \& Prevor, 2000). Enquanto o paradigma do "olhar preferencial" é uma tarefa de reconhecimento, comparando-se um evento visual anterior com um atual, e fornece uma medida indireta sobre "quantos objetos o bebê acha que tem naquele evento", a busca manual é uma tarefa com maiores demandas de memória de trabalho, uma vez que não há comparação e exige a manutenção da representação dos objetos por 10 a $20 \mathrm{seg}$, fornecendo, portanto, uma evidência direta, representada pela busca de um objeto que está na mente do bebê (Van de Walle et al., 2000). A busca manual por objetos escondidos tem produzido resultados semelhantes e até mais contundentes que os do paradigma do "olhar preferencial", revelando que, de fato, os mecanismos cognitivos envolvidos em ambos os métodos são os mesmos. Ao verem um experimentador esconder uma bolacha num balde opaco à esquerda e, depois, esconder sucessivamente $1+1=2$ bolachas no balde à direita, bebês de 10 a 12 meses procuram o balde com a maior quantidade e fazem o mesmo com arranjos 1 vs. 3, 2 vs. 3, mas falham ao comparar 2 vs. 4, 
3 vs. 4 e 3 vs. 6 (Feigenson, Carey, \& Hauser, 2002). Entretanto, além da numerosidade per se, este sistema numérico exato também computa as propriedades contínuas de pequenas coleções subitizáveis. No mesmo estudo com biscoitos realizado por Feigenson et al. (2002), quando os experimentadores colocaram um biscoito com o dobro do tamanho num balde e dois biscoitos menores no outro balde cujas áreas somadas era a metade do biscoito grande, os bebês escolheram o balde com o biscoito maior e não o balde com os dois biscoitos menores, portanto, baseando sua escolha nas informações quantitativas contínuas e não-numéricas dos objetos. Estudos posteriores com o paradigma da busca manual mostraram que quando essas variáveis contínuas são totalmente controladas, de forma a criar coleções subitizáveis numericamente diferentes mantendo-se constante a área dos objetos escondidos, os bebês sempre baseiam suas buscas no número (Feigenson \& Carey, 2003).

\section{Senso Numérico: Dois Sistemas Distintos, o Exato e o Aproximado}

Juntas, as abordagens de habituação (Carey, 2002; Wynn, 1992a) e do alcance manual dos objetos (Feigenson \& Carey, 2003; Van de Walle et al., 2000) revelam que o comportamento numérico dos bebês é sólido e não apenas baseado nas propriedades visuais contínuas e não-numéricas dos estímulos e que, além de rastrear mentalmente os objetos, eles também podem representar as propriedades genuinamente numéricas dos estímulos. O comportamento numérico dos bebês apresenta algumas características típicas, isto é, "assinaturas" ou "rubricas" comportamentais que aparecem independentemente do paradigma experimental usado.

Também ficou claro que a representação numérica súbita, sem contagem e sem o uso da linguagem, é limitada a pequenas quantidades, denominadas numerosidades subitizáveis (Starkey \& Cooper, 1980) de até no máximo 3 objetos em bebês e aproximadamente 4 objetos em adultos (Mandler \& Shebo, 1982; Trick \& Pylyshyn, 1994), e que este sistema numérico exato ou "subitizador" evoluiu não somente para o rastreamento preciso de um pequeno número de indivíduos, mas também para a representação de informações sobre suas propriedades quantitativas contínuas. É fácil entender a evolução biológica dessas capacidades se compreendermos que um predador, por exemplo, necessita saber não só o número de indivíduos no grupo que ele pretende atacar, mas também se a presa alvo é a menor e, portanto, a mais fácil de ser abatida, ou, ainda, se ele faz parte de um grupo que se encontra em número suficiente para abater uma grande presa.

Por outro lado, há muito tempo sustenta-se que há um sistema numérico puro que permite a representação numérica aproximada de grandes coleções, baseada exclusivamente nas propriedades numéricas, que está presente em animais e bebês humanos (Dehaene, 1997). Experimentos recentes com o paradigma da habituação, que controlam totalmente as informações de quantidades contínuas e não-numéricas, revelam que bebês de 6 meses de idade discriminam entre arranjos numéricos grandes, acima de 3 , que diferem na razão $1: 2$, tais como 8 e 16,16 e 32 pontos, mas falham em razões muito menores de 1:1,5, como 8 e 12, ou 16 e 24 pontos. Assim, estas representações para numerosidades maiores, as únicas exclusivamente numéricas, uma vez que o sistema numérico exato da subitização também computa quantidades contínuas, são sempre aproximadas, mas cuja precisão aumenta com o desenvolvimento. A relação entre as coleções tem um limite mínimo de 1:2 nos bebês de 6 meses, de 2:3 (ou 1:1,5) em bebês de 10 meses (Lipton \& Spelke, 2003; Xu \& Spelke, 2000) e pode ser tão próxima quanto $7: 8$ em adultos (Barth, Kanwisher, \& Spelke, 2003). Finalmente, assim como na subitização, as representações numéricas aproximadas não são restritas à modalidade visual e se estendem para sequências de eventos temporalmente distintos tais como sons e com as mesmas assinaturas comportamentais observadas com estímulos visuais, isto é, com os padrões de sucesso e falhas relacionadas às proporções entre as coleções (Lipton \& Spelke, 2004).

Em suma, hoje sabemos que primatas não-humanos, bebês e adultos humanos possuem 
dois sistemas numéricos de natureza supramodal, sensíveis tanto a objetos quanto a eventos como pulos de um fantoche (Dehaene et al., 1998; Feigenson et al., 2004; Hauser \& Spelke, 2004; Hauser, Tsao, Garcia, \& Spelke, 2003). Um é o sistema numérico exato, para quantidades de até 3 objetos, o qual também computa variáveis contínuas, chamado de sistema subitizador ou de rastreamento de objetos (Trick \& Pylyshyn, 1994). Esse sistema subitizador serve para rastrear indivíduos no espaço e no tempo, mas não parece ter evoluído especificamente para enumerar objetos ou realizar comparações numéricas e, embora macacos e humanos possam usá-lo para representar os objetos como indivíduos, não o usam especificamente para representá-los como um grupo com valor cardinal (Xu, Spelke, \& Goddard, 2005). O outro, o sistema numérico aproximado, para quantidades maiores que 3 , tem se revelado como especificamente numérico, ou seja, é imune às variáveis não numéricas.

Experimentos com animais, usando paradigmas semelhantes aos aqui reportados, revelaram que pequenos primatas possuem as mesmas capacidades numéricas com as mesmas assinaturas comportamentais observadas em bebês e adultos humanos (Hauser et al., 2003). Interessante, embora as habilidades numéricas espontâneas em macacos sem treinamento proporcionem respostas mais lentas na discriminação numérica aproximada do que em macacos treinados, ainda assim eles são capazes de discriminar coleções com proporções entre 1,25 e 1,5 (Hauser et al., 2003), superando em muito os bebês de 6 meses com arranjos e métodos muito similares (Lipton \& Spelke, 2003).

Outras investigações psicológicas sobre a cognição numérica demonstram que as representações numéricas e o pensamento matemático culturalmente construído dependem, em parte, de um senso aproximado de magnitudes numéricas, ou seja, um "senso numérico" de natureza não verbal (Dehaene, 1997; Gallistel \& Gelman, 1992). Quando se impede os adultos de contarem, eles ainda são aptos a determinarem a quantidade exata de pequenas quantidades (subitizáveis), mas somente a quantidade aproximada de numerosidades grandes (não subitizáveis;
Cordes, Gelman, Gallistel, \& Whalen, 2001). Mesmo quando os números são simbolicamente expressos em algarismos especificando a quantidade exata, ainda assim estes símbolos evocam um senso numérico aproximado com o qual raciocinamos quando comparamos números ou fazemos subtrações ou somas de números muito grandes (Dehaene, 1997). É por isso que os adultos são mais rápidos em dizer qual o maior de dois números relativamente distantes (por exemplo: 9 e 5) do que de dois números muito próximos (5 e 6; Dehaene, Dupoux, \& Mehler, 1990), bem como mais rápidos em rejeitar respostas erradas de problemas aritméticos quando o número errado é muito distante do correto (Pinel, Dehaene, Riviere, \& LeBihan, 2001).

Finalmente, resultados de estudos antropoculturais mostram que o sistema empobrecido de contagem em culturas indígenas da Amazônia, como os Pirahã, que usam um sistema de contagem um-dois-muitos, e os Mundurucu, que possuem palavras-número apenas até "cinco", limita sua habilidade para enumerar quantidades exatas em arranjos numéricos grandes, acima de dois ou três itens nos Pirahã (Gordon, 2004) e acima de cinco itens nos Mundurucu (Pica, Lemer, Izard, \& Dehaene, 2004). Entretanto, ambas as culturas possuem uma habilidade numérica de quantificação não verbal (sem contagem), uma exata para pequenas quantidades (até 3) e outra aproximada para grandes quantidades, semelhantes à dos adultos enculturados da sociedade moderna, as quais parecem se originar dos mesmos mecanismos cognitivos subjacentes à subitização e à quantificação aproximada (Gelman \& Butterworth, 2005), os mesmos já constatados em animais e bebês (Hauser \& Spelke, 2004; Spelke, 2003).

Juntas, as evidências antropoculturais, psicológicas e desenvolvimentais apontam para a universalidade de dois sistemas numéricos inatos não verbais: um sistema exato para pequenas quantidades até 3 ou 4 itens e um sistema aproximado para grandes quantidades, além de um sistema numérico exato de natureza verbal, culturalmente determinado. Adultos, bebês, crianças pré-escolares e primatas não-humanos parecem compartilhar um sistema de processamento nu- 
mérico aproximado para estímulos não simbólicos tais como coleções de pontos ou sequências de tons. Estudos comportamentais de humanos adultos implicam uma ligação entre estas habilidades numéricas não-simbólicas e processamento numérico simbólico (e.g. similares efeitos da distância na precisão e no tempo de reação para arranjos de pontos e numerais arábicos).

\section{Os Circuitos Neurais do Comportamento Numérico}

As "assinaturas comportamentais" de um senso numérico básico são corroboradas por evidências de uma "assinatura neurológica" que revelam a existência de substratos neurais evolutivamente determinados do comportamento numérico. Cinco tipos de evidências neurológicas suportam a ideia de que porções bilaterais do córtex parietal inferior, particularmente a porção horizontal do sulco intraparietal, desempenham um papel crucial na representação numérica. Vejamos, então, um resumo da neurocognição numérica a partir de um enfoque histórico.

\section{A Neuropsicologia dos Números: Estudos de Lesão Cerebral}

No início do século XX, Lewandowsky e Stadelmann (1908), forneceram o primeiro relato detalhado de um paciente cujos danos focais em áreas visuais do cérebro induziram distúrbios seletivos do cálculo. O paciente podia fazer cálculos mentais, mas tinha severas dificuldades com a leitura dos símbolos aritméticos. Esse estudo constituiu um marco sobre a neuropsicologia e a neurocognição da matemática, por produzir evidência de que desordens de cálculo podem ser diferentes e dissociadas dos distúrbios de linguagem (Ardila \& Rosselli, 2002).

Ainda em 1919, o neurologista sueco Solomon E. Henschen (1847-1930), com grande interesse nas afasias e no processamento visual, descobriu que danos focais no córtex parietal prejudicavam, com relativa seletividade, o cálculo matemático (Henschen, 1919). Henschen revisou 305 casos da literatura além dos estudos com 67 de seus pacientes e confirmou que lesões focais em certas áreas cerebrais do hemisfério esquerdo, próximas, mas distintas daquelas envolvidas na linguagem, prejudicavam cálculos matemáticos, preservando a linguagem e a música. Henschen reportou que a terceira convolução (giro) do córtex frontal inferior corresponderia ao centro da pronúncia dos números, ao passo que duas áreas posteriores no córtex parietal inferior, particularmente no giro angular (área de Brodman BA39) estariam envolvidas mais especificamente no processamento numérico: a parte mais ínfero-posterior do giro angular (mais próxima dos córtices visuais no giro occipital) seria o centro da "escrita dos números", ao passo que a área mais superior do giro angular, envolvendo a fissura intraparietal, seria o centro da "leitura dos números". Henschen foi quem cunhou o termo "acalculia", usado até hoje na neuropsicologia. Assim, os estudos de Henschen sugeriram sistemas neurais independentes que pareciam especialmente engajados em aspectos específicos na aritmética básica e estabeleceram de uma vez por todas as bases das futuras pesquisas neurológicas sobre processamento dos números (Ardila $\&$ Rosseli, 2002)

O neurologista alemão Hans Berger (18731941), responsável pelo primeiro uso da técnica de eletroencefalograma (EEG) em humanos, também foi o primeiro a introduzir a distinção entre acalculia primária e secundária (H. Berger, 1926). Conforme H. Berger, a acalculia primária ou pura se caracterizaria pela perda de conceitos numéricos e da habilidade de compreender e executar operações aritméticas básicas, também cunhada de "anarimetia", ao passo que a acalculia secundária compreenderia déficits no cálculo decorrentes de outros déficits cognitivos não específicos dos números, isto é, de déficits de domínio mais geral, tais como a memória, a linguagem, etc. Portanto, os estudos de H. Berger e sua nomenclatura proposta, representam a primeira distinção clara entre déficits de cálculo de natureza mais especificamente numérica e déficits de cálculo decorrentes de prejuízos cognitivos na linguagem ou na memória (Ardila \& Rosseli, 2002).

Finalmente, o neurologista austríaco Josef Gerstmann (1887-1969) propôs, com base em 
inúmeros estudos de prejuízos na cognição matemática decorrentes de danos cerebrais, que a acalculia primária resultaria de lesões no giro angular esquerdo e estaria sistematicamente associada com a agrafia, desorientação espacial esquerda-direita e agnosia digital (Gerstmann, 1940). A acalculia primária, juntamente com este conjunto de sintomas não matemáticos, constitui uma única síndrome que, desde então, é chamada de "síndrome de Gerstmann" e faz parte do repertório de avaliações neuropsicológicas até os dias de hoje (Ardila \& Rosselli, 2002; Dehaene \& Cohen, 1997).

Em 1961, Hécaen, Angelerges, e Houllier publicaram um extenso trabalho que investigou 183 pacientes com lesões exclusivamente nas áreas posteriores à fissura de Rolando. Os pesquisadores identificaram três tipos principais de desordens de cálculo: (a) alexia e agrafia para números, associada ou não com palavras; (b) acalculia espacial, desordem da organização espacial dos dígitos que leva a erros de cálculo, mas com preservação dos números e dos fatos aritméticos (frequentemente associada com heminegligência espacial e inversões de números); (c) anaritmetia (acalculia primária), déficit básico na habilidade matemática computacional, que parece refletir déficits conceituais para números, excluindo a alexia e a agrafia para números $\mathrm{e}$ acalculia espacial. Entretanto, assim como todos os outros autores reportados, Hécaen e seus colegas (1961) não supõem um déficit isolado dos conceitos numéricos e operações aritméticas. É importante notar também que, ao proporem que a anaritmetia pode ser encontrada sem estar associada à agrafia para números e acalculia espacial, Hécaen et al. (1961) fornecem uma descrição de acalculia primária mais seletiva do que a síndrome de Gerstmann.

Resumindo esta pequena incursão histórica dos déficits matemáticos em estudos de lesão cerebral, podemos concluir que a anaritmetia (ou acalculia primária) é um déficit básico da habilidade computacional dos números. A acalculia primária corresponde a uma incapacidade de compreender quantidades e fazer comparações e estimações numéricas, isto é, perda de conceitos numéricos e uso de regras de cálculo para exe- cutar operações aritméticas básicas. Os déficits de cálculo na anaritmetia são encontrados em operações escritas e orais, com preservação da linguagem, conhecimento dos números, da contagem e outros fatos aritméticos memorizados verbalmente. Portanto, tornou-se largamente conhecido e aceito na neurologia que a anaritmetia está associada a danos no giro angular esquerdo do lobo parietal desde os estudos de Henschen e representa danos a um sistema conceitual genuinamente numérico que independe da linguagem. Em suma, embora danos linguísticos normalmente causem sérios prejuízos à aritmética, danos em áreas não linguísticas podem causar prejuízos de igual seriedade em aspectos conceituais básicos dos números, os quais desencadeiam prejuízos no cálculo independentes da preservação da linguagem.

Juntando-se esses achados históricos aos estudos neuropsicológicos mais recentes, podemos resumir a neurocognição dos números nos parágrafos que se seguem.

Primeiro, lesões parietais em adultos podem causar danos seletivos da compreensão e operações com números, preservando a linguagem (Dehaene \& Cohen, 1997; Delazer, Girelli, Semenza, \& Denes, 1999; Lemer, Dehaene, Spelke, \& Cohen, 2003). Reciprocamente, o número pode ser seletivamente preservado na presença de severos déficits no processamento de outras categorias de palavras em pacientes com lesões parietais e linguagem preservada (Dehaene \& Cohen, 1997). São casos em que, apesar de não ser capaz de dizer qual o número médio entre 1 e 3, por exemplo, o paciente consegue realizar esse tipo de tarefa com letras, dias da semana, meses, ou notas de uma escala musical. Mais intrigante ainda é um estudo recente de três pacientes com extensa lesão nas áreas linguísticas perissilvianas e grave quadro de afasia, incluindo afasia de expressão, severo agramatismo tanto na linguagem oral quanto escrita e apenas uma pequena compreensão léxica, que ainda assim preservaram a matemática até mesmo mais complexa, incluindo operações com dois e três dígitos e operações com parênteses do tipo (3 x 3) - 6 (Varley, Klessinger, Romanowski, \& Siegal, 2005). 
Segundo, comparações entre as bases cerebrais do número em culturas diferentes indicam que o envolvimento do sulco intraparietal é, de fato, universal. Se a matemática fosse uma atividade exclusivamente cultural envolvendo uma arquitetura cerebral eminentemente cultural, seria de se esperar uma considerável variação das áreas cerebrais envolvidas em função do aprendizado, educação e cultura. Relatórios clínicos de vários lugares do mundo, entretanto, confirmam que as lesões que ocasionam a discalculia adquirida ou acalculia (perda do senso numérico), assim como as áreas de ativação neural durante tarefas numéricas em indivíduos sadios, estão sistematicamente associadas à região parietal inferior (Dehaene et al., 1998).

Terceiro, estudos sobre a discalculia do desenvolvimento (um déficit congênito específico na percepção numérica e no aprendizado da matemática escolar, que afeta de $5 \%$ a $6 \%$ das crianças que, a despeito de um QI completamente normal, aquisição normal da leitura e escrita e da escolaridade, nunca adquirirão o conceito de número), indicam contribuição altamente específica do sulco intraparietal para o processamento do número (Butterworth, 1999; Temple, 1989). Elas precisam confiar em estratégias verbais laboriosas mesmo em tarefas tão simples como determinar que nove é maior do que três, ou que um pato possui duas pernas (Butterworth, 1999).

\section{A Neurocognição Numérica em Estudos de Neuroimagem}

Estudos recentes de neuroimagem (Dehaene, Piazza, Pinel, \& Cohen, 2003) e técnicas neurofisiológicas utilizando EEG (Dehaene, 1996; Kiefer \& Dehaene, 1997) mostram que o segmento horizontal do sulco intraparietal, bilateralmente (Dehaene et al., 2003), é a área de maior ativação em indivíduos sadios enquanto desempenham vários tipos de tarefas numéricas simbólicas, como comparação numérica (qual de dois números é o maior), aproximação numérica e operações aritméticas básicas mais complexas de dois dígitos (Hubbard, Piazza, Pinel, \& Dehaene, 2005). O sulco intraparietal em ambos os hemisférios é uma área de integração multimodal espaço-temporal das informações visuais, auditivas e motoras, e na cognição matemática esta área está envolvida na convergência multimodal da entrada de informação simbólica e não-simbólica (processamento perceptivo dos objetos e seus atributos espaço-temporais) no processamento matemático (Feigenson et al., 2004). O envolvimento do sulco intraparietal nos conceitos numéricos básicos, tanto nos estudos de lesão quanto nos de neuroimagem, reforçam os achados psicológicos no sentido de que, não obstante a participação crucial da linguagem na aritmética exata, o conceito numérico básico é um sistema cognitivo não linguístico de natureza espaço-temporal e, portanto, supramodal (Hubbard et al., 2005).

Cantlon, Brannon, Carter e Pelphrey (2006), usando a ressonância magnética funcional (fMRI) em crianças de 4 anos de idade e adultos enquanto estes observaram arranjos de elementos que podiam variar tanto no número quanto apenas na forma local dos elementos, mostraram que ambos, crianças e adultos, apresentaram uma maior resposta do sulco intraparietal para arranjos visuais que desviavam do estímulo padrão no seu número de elementos do que para estímulos que desviavam na forma local do elemento. Esta é a primeira evidência de que os circuitos neurais da cognição numérica conhecidos no adulto já ocorrem desde muito cedo no desenvolvimento, antes da experiência simbólica sofisticada, em consonância com as evidências comportamentais aqui reportadas e discutidas.

Replicando, com alterações, o procedimento de Wynn (1992a) em combinação com a técnica de potenciais relacionados a eventos (PRE), A. Berger e colegas (2006) mediram a ativação eletrofisiológica diferencial no escalpo de bebês de 6 a 9 meses enquanto estes viam equações aritméticas simples com resultados corretos e incorretos. Estas ativações foram comparadas com as de adultos observando equações matemáticas simbólicas corretas e incorretas e os resultados mostraram que além de os bebês terem olhado por menos tempo para as equações corretas do que para as incorretas, como esperado, seu cérebro apresentou potenciais negativos em 
sincronia com as apresentações da solução significantemente diferente para as equações incorretas. Mais interessante ainda é que o padrão de ativações no cérebro dos bebês foi semelhante ao dos adultos.

Finalmente, um estudo ainda mais recente realizado por Izard, Dehaene-Lambertz, e Dehaene (2008), produziu resultados bastante interessantes. É sabido que o cérebro humano possui áreas diferentes para o processamento dos atributos de identidade dos objetos (cor e forma), localizadas ventralmente nos córtices occipito-temporais inferiores, ao passo que o processamento visuo-espacial e de movimento é servido por um sistema dorsal nas áreas occipito-parietais superiores (Andrade, 2006a; Lent, 2001). Conforme também vimos, o processamento numérico, de natureza espaço temporal é dependente de uma área específica deste sistema dorsal, particularmente o sulco intraparietal bilateralmente (Dehaene et al., 2003). Izard et al. (2008) registraram os potenciais elétricos em bebês de apenas 3 meses de idade, evocados tanto por mudança na identidade dos objetos quanto na sua cardinalidade em um dado arranjo observado. Usando um modelo 3-D da cabeça do bebê, Izard e colegas (2008) reconstruíram as fontes corticais destas respostas eletrofisiológicas e da mesma forma que nos adultos as mudanças de identidade do objeto e de número foram distintas, revelando uma organização básica ventral/dorsal já definida no cérebro dos bebês. Como nos adultos, a identidade do objeto nos bebês é codificada ao longo de um circuito ventral nos lobos temporais, ao passo que as mudanças de numerosidade ativaram uma rede parietoprefrontal, mas principalmente no hemisfério direito. Estes resultados não somente enfatizam a continuidade desenvolvimental do senso numérico como também apontam para uma propensão funcional inata na organização cerebral.

Finalmente, muitas espécies animais respondem à numerosidade, tanto em experimentos usando o procedimento de "escolha de acordo com o modelo" (matching to sample) quanto espontaneamente, em experimentos de busca manual por alimentos humanos (Hauser \& Spelke,
2004; Hauser et al., 2003) semelhantes àqueles desenvolvidos com bebês (Feigenson \& Carey, 2003). Vários estudos já existem que usam registros neurofisiológicos de populações neuronais utilizando múltiplos eletrodos diretamente no cérebro de macacos, revelaram um sistema parieto-frontal seletivamente ativado pelos números, particularmente nas vizinhanças do sulco intraparietal em uma área homóloga ao do sulco intraparietal do cérebro dos humanos (para uma revisão, veja Nieder, 2005).

\section{Considerações Finais}

Todos os humanos, independentemente de sua cultura e educação, possuem uma compreensão intuitiva de número (Dehaene, 1997). Nas últimas décadas, a investigação sistemática dos precursores das habilidades numéricas em animais e em bebês e adultos humanos tem lançado luzes sobre as origens da aritmética culturalmente construída. Pesquisas tem revelado que animais como ratos, pombos e macacos podem extrair a numerosidade aproximada de grupos de objetos visuais e auditivos. A numerosidade é representada pelos animais independentemente de outros parâmetros, tais como tamanho ou forma do objeto (Hauser \& Spelke, 2004; para uma breve revisão em português veja Andrade, 2006a, 2006b e Prado, 2010). Evidências comportamentais, de imagem cerebral e neurofisiológicas, produzidas em pesquisas com primatas, bebês e adultos humanos, convergem no sentido de que o conhecimento do número é fruto do processo evolutivo e foi herdado de ancestrais filogenéticos, cuja evolução configurou uma base cortical no córtex intraparietal, bilateralmente (Feigenson et al., 2004; Hubbard et al., 2005). A hipótese do senso numérico postula que este sistema cerebral já está disponível desde muito cedo no desenvolvimento, em bebês tão novos quanto três meses de idade e guia o aprendizado dos numerais e da aritmética na infância e a aquisição da matemática complexa adulta. As evidências comportamentais e neurológicas deste sistema numérico não podem ser atribuídas a uma reação atencional de domínio geral à novidade ou à familiaridade. 
As evidências não suportam o logicismo piagetiano, no qual o conceito de número é o resultado de um longo período de construções lógicas sensório-motoras, consolidando-se somente após os 6-7 anos de idade. Tampouco elas suportam o logicismo proposicional de Vygotsky, no qual o conceito de número também surge com as generalizações complexas dependente da linguagem. Ao contrário, as evidências ressaltam a continuidade entre filogênese e ontogênese do senso numérico apontando para tendências funcionais na organização cerebral que podem canalizar para áreas restritas do cérebro o aprendizado subsequente. Este senso numérico inato, entretanto, é baseado em dois mecanismos cognitivos numéricos supramodais, isto é, respondem a números em qualquer modalidade sensorial (auditiva ou visual, com estímulos estáticos ou móveis, etc.). Um sistema é exato, mas limitado à apreensão súbita de 3 ou 4 elementos, chamado "subitização". O outro é aproximado, para numerosidades maiores. Assim, ambos os sistemas são muito limitados e estão longe da aritmética exata para grandes numerosidades e mais distantes ainda da matemática complexa culturalmente construída.

A existência de um senso numérico de natureza perceptiva espaço-temporal é consistente em parte com a posição de Piaget de que a linguagem não era tudo. Entretanto, em crianças mais velhas e adultos este sistema numérico de natureza não-verbal é suplementado pela aquisição da linguagem, como os símbolos para quantidades, as rotinas de cálculo (algoritmos), etc., que possibilitam a aprendizagem da matemática culturalmente construída.

Os recursos que a linguagem possibilita têm sido implicados na cognição matemática de diversas formas (Spelke, 2003). Uma delas é que as palavras-número fornecem uma base para se aprender a manipular, com precisão, quantidades grandes que só podem ser apreendidas de forma aproximada pela percepção numérica (Bloom, 2000). Outra forma, esta bastante óbvia, é a linguagem poder representar um código no qual as computações matemáticas são realizadas (Carey, 2004; Spelke, 2003). Estes papéis da linguagem para a matemática exata e mais sofisticada parecem ficar evidentes em culturas nas quais a ausência de palavras-número parece resultar na limitação da cognição numérica à enumeração exata até 3 objetos e enumeração aproximada em arranjos acima de 3 (Gordon, 2004; Pica et al., 2004). Há também os que argumentam que existem fortes paralelos entre a sintaxe da linguagem natural e a estrutura da Matemática (Hauser, Chomsky, \& Fitch, 2002). Uma discussão detalhada das evidências e propostas mais recentes da cognição numérica foge ao escopo desta revisão, merecendo um artigo específico.

Em um estudo com crianças entre 2 a 2,5 anos, Wynn (1992b) produziu resultados extremamente esclarecedores sobre pelo menos um dos aspectos da integração entre os sistemas do senso numérico inato e a linguagem. Ao usar uma tarefa numérica, a qual ela chamou de "dê um número", as crianças eram requisitadas a dar a um fantoche (que falava com ela) 1 a 6 itens de uma pilha de animais de brinquedo. Mesmo as crianças mais novas (2,5 anos) deram um objeto quando solicitadas a dar um objeto, ao passo que nenhuma delas deu um objeto quando solicitada a dar dois, três ou quatro objetos, mostrando uma clara compreensão de que as palavras-número indicam numerosidades. Entretanto, a despeito deste conhecimento inicial, as crianças levaram aproximadamente um ano inteiro a mais para aprender quais palavras se referem a quais numerosidades. Wynn (1992b) encontrou que uma criança de 2,5 anos pode, de fato, conhecer a cardinalidade de pequenos conjuntos subitizáveis de itens como, por exemplo, que 3 é mais que 2 e menos que 4 sem saber, necessariamente, que o último número de um contagem indica a numerosidade (a cardinalidade). As crianças mais novas (2,5 anos), mesmo que ainda estivessem começando a aprender a contar, foram bem sucedidas quando requeridas a dar apenas um brinquedo ao fantoche, bem sucedidas algumas vezes quando requeridas a dar 2 animais, e quase sempre deram um punhado de brinquedos ao serem requisitadas a dar de três a cinco animais, quase nunca recorrendo à contagem. Por outro lado, as crianças de 3,5 anos tenderam a contar 
os itens de uma pilha à medida que os davam ao fantoche e sempre paravam na palavra número pedida, e foram bem sucedidas até à numerosidade de três ou quatro. Em suma, Wynn (1992b) mostrou que por volta de 3,5 anos de idade as crianças já imputam um significado às palavras-número que de alguma forma está ligada à numerosidade, isto é, à cardinalidade, quando esta está dentro do seu alcance de contagem.

As evidências sugerem, portanto, uma confluência entre os dois sistemas cognitivos inatos que constituem o senso numérico e a linguagem, incluindo a contagem e o uso dos algoritmos no aprendizado da aritmética exata.

Finalmente, o fato de o comportamento matemático ser crucialmente dependente do senso numérico e este, por sua vez, ser uma capacidade inata servida por um substrato neural relativamente específico, isto implica na possibilidade de um prejuízo relativamente específico do desenvolvimento normal das competências numéricas com preservação da linguagem. Realmente, alterações congênitas sutis envolvendo a porção horizontal do sulco intraparietal estão sistematicamente associadas à discalculia do desenvolvimento com preservação da inteligência geral e de outros domínios cognitivos.

Da discussão anterior, decorre que a não admissão da natureza inata e neurobiológica das operações mentais subjacentes aos domínios culturalmente construídos e seu aprendizado implica na negação de qualquer dificuldade de aprendizagem de ordem congênita, exceto nos casos de lesões (Andrade, 2006a) ou deficiência intelectual. As dificuldades de aprendizagem são, pois, vistas como determinadas exclusivamente, com poucas exceções, por insuficiências no processo de ensino. É óbvio que este é de fundamental importância e sua relação causal com o desempenho dos alunos não pode, absolutamente, ser descartada. Porém, pesquisas como as que acabamos de resumir fornecem bases bastante consistentes para a forja de uma visão que supera a falsa dicotomia entre biologia e cultura, as quais passam a ser vistas como continuidade uma da outra.

Dificuldades de aprendizagem são multideterminadas e isso inclui a dimensão biológi- ca. Como é mostrado por uma vasta literatura calcada em dados empíricos produzidos por pesquisas conduzidas com todo rigor científico, há amplo consenso de que uma parcela da população com acesso a boas oportunidades de ensino, inteligência normal e ausência de quaisquer comprometimentos sensoriais, sofre com transtornos persistentes, os quais impõem barreiras quase insuperáveis para o aprendizado da matemática, assim como da leitura, no caso da dislexia. Em se tratando, pois, de dificuldades tão específicas, são necessários instrumentos especialmente desenvolvidos para que possam ser detectadas. Da mesma forma, é indispensável que disponhamos de métodos de ensino elaborados e testados cientificamente. E além desses recursos, é absolutamente imprescindível que a formação de educadores seja repensada, de modo a assegurar-lhes capacitação técnica e científica para que sua ação pedagógica seja verdadeiramente inclusiva.

\section{Referências}

Aguiar, A., \& Baillargeon, R. (1999). 2.5-month-old infants' reasoning about when objects should and should not be occluded. Cognitive Psychology, 39, 116-157. doi:10.1006/cogp.1999.0717

Andrade, P. E. (2004). Uma abordagem evolucionária e neurocientífica da música. Neurociências, 1, 21-33.

Andrade, P. E. (2006a). A teoria sócio-culturalista de Vygotsky e o papel da linguagem na formação de conceitos: O que a psicologia experimental e a neurociência têm a nos dizer. Neurociências, $3,158-178$

Andrade, P. E. (2006b). O desenvolvimento cognitivo da criança: $\mathrm{O}$ que a psicologia experimental e a neurociência têm a nos dizer. Neurociências, 3, 98-118.

Andrade, P. E., \& Prado, P. S. T. (2003). Psicologia e neurociência cognitivas: Alguns avanços recentes e implicações para a educação. Interação em Psicologia, 7(2), 73-80. doi:10.5380/psi. v7i2.3225

Ardila, A., \& Rosselli, M. (2002). Acalculia e dyscalculia. Neuropsychological Review, 12, 179-231. doi:10.1177/1367006912466313 
Barth, H., Kanwisher, N., \& Spelke, E. (2003). The construction of large number representations in adults. Cognition, 86(3), 201-221. doi:10.1016/ s0010-0277(02)00178-6

Berger, A., Tzur, G., \& Posner, M. I. (2006). Infant brains detect arithmetic errors. Proceedings of the National Academy of Science, 103(33), 12649-12653. doi:10.1073/pnas.0605350103

Berger, H. (1926). Uber Rechenstorunger bei Herderkraunkunger des Grosshirns. Archives für Psychiatrie und Nervenkrankheiten, 78, 236-263.

Bloom, P. (2000). How children learn the meaning of words. Cambridge, MA: Massachusetts Institute of Technology Press.

Butterworth, B. (1999). The mathematical brain. London: Macmillan.

Cantlon, J. F., Brannon, E. M., Carter, E. J., \& Pelphrey, K. A. (2006). Functional imaging of numerical processing in adults and 4-y-old children. Public Library of Science- Biology, 4(5), e125. doi:10.1371/journal.pbio.0040125

Carey, S. (1997). Do constraints on word meaning reflect prelinguistic cognitive architecture? The Japanese Journal of Cognitive Science, 4(1), 35 58. doi:10.11225/jcss.4.1_35

Carey, S. (2002). Evidence for numerical abilities in young infants: A fatal flaw? Developmental Science, 5(2), 202-205. doi:10.1111/14677687.00221_1

Carey, S. (2004). Bootstrapping \& The origins of concepts. Daedalus, 5, 59-68. doi:10.1162/001152604772746701

Cohen, L. B., \& Marks, K. S. (2002). How infants process addition and subtraction events. Developmental Science, 5, 186-201. doi:10.1111/14677687.00220

Cordes, S., Gelman, R., Gallistel, C. R., \& Whalen, J. (2001). Variability signatures distinguish verbal from nonverbal counting for both small and large numbers. Psychonomic Bulletin \& Review, 8, 698-707. doi:10.3758/BF03196206

Dehaene, S. (1996). The organization of brain activations in number comparison: Event-related potentials and the additive-factors method. Journal of Cognitive Neuroscience, 8, 47-68. doi:10.1162/jocn.1996.8.1.47

Dehaene, S. (1997). The number sense. New York: Oxford University Press.
Dehaene, S., \& Cohen, L. (1997). Cerebral pathways for calculation: Double dissociation between rote verbal and quantitative knowledge of arithmetic. Cortex, 33(2), 219-250. doi:10.1016/ S0010-9452(08)70002-9

Dehaene, S., Dehaene-Lambertz, G., \& Cohen, L. (1998). Abstract representations of numbers in the animal and human brain. Trends in Neuroscience, 21(8), 355-361. doi:10.1016/S01662236(98)01263-6

Dehaene, S., Dupoux, E., \& Mehler, J. (1990). Is numerical comparison digital? Analogical and symbolic effects in two-digit number comparison. Journal of Experimental Psychology: Human Perception \& Performance, 16, 626-641. doi:10.1037/0096-1523.16.3.626

Dehaene, S., Piazza, M., Pinel, P., \& Cohen, L. (2003). Three parietal circuits for number processing. Cognitive Neuropsychology, 20, 487506. doi:10.1080/02643290244000239

Delazer, M., Girelli, L., Semenza, C., \& Denes, G. (1999). Numerical skills and aphasia. Journal of the International Neuropsychology Society, 5(3), 213-221. doi:10.1017/S1355617799533043

Fantz, R. L. (1964). Visual experiences in infants: Decreased attention to familiar patterns relative to novel ones. Science, 146, 668-670. doi:10.1126/ science. 146.3644 .668

Feigenson, L., \& Carey, S. (2003). Tracking individuals via object files: Evidence from infants' manual search. Developmental Science, 6(5), 568-584. doi:10.1111/1467-7687.00313

Feigenson, L., Carey, S., \& Hauser, M. (2002). The representations underlying infants' choice of more: Object files vs. analog magnitudes. Psychological Science, 13, 150-156.

Feigenson, L., Dehaene, S., \& Spelke, E.S. (2004). Core systems of number. Trends in Cognitive Sciences, 7, 307-314. doi:10.1016/j. tics.2004.05.002

Gallistel, C. R., \& Gelman, R. (1992). Preverbal and verbal counting and computation. Cognition, 44, 43-74. doi:10.1016/0010-0277(92)90050-R

Gelman, R., \& Butterworth, B. (2005). Number and language: How are they related? Trends in Cognitive Science, 9, 6-10. doi:10.1016/j. tics.2004.11.004

Gerstmann, J. (1940). The syndrome of finger agnosia, disorientation for right and left, agraphia and 
acalculia. Archives of Neurology and Psychiatry, 44, 398-408.

Gordon, P. (2004). Numerical cognition without words: Evidence from Amazonia. Science, 306(5695), 496-499. doi:10.1126/science. 1094492

Hauser, M. D., Chomsky, N., \& Fitch, W. T. (2002). The faculty of language: What is it, who has it, and how did it evolve? Science, 298(5598), 1569-1579. doi:10.1126/science.298.5598.1569

Hauser, M. D., \& Spelke, E. S. (2004). Evolutionary and developmental foundations of human knowledge: A case study of mathematics. In M. Gazzaniga (Ed.), The cognitive neurosciences (pp. 853-864). Cambridge, MA: Massachusetts Institute of Technology Press.

Hauser, M. D., Tsao, F., Garcia, P., \& Spelke, E. S. (2003). Evolutionary foundations of number: Spontaneous representation of numerical magnitudes by cotton-top tamarins. Proceedings of the Royal Society B: Biological Sciences, 270, 1441-1446. doi:10.1098/rspb.2003.2414

Hécaen, H., Angelerges, T., \& Houllier, S. (1961). Les varietes cliniques des acalculies au cours des lésions retro-rolandiques: approche statistique du pròbleme. Revue Neurologique, 105, 85-103.

Henschen, S. E. (1919). Clinical and anatomical contributions on brain pathology. Archives of Neurology and Psychiatry, 13, 226-249.

Hubbard, E. M., Piazza, M., Pinel, P., \& Dehaene, S. (2005). Interactions between number and space in parietal cortex. Nature Reviews Neuroscience, 6(6), 435-448. doi:10.1038/nrn1684

Huntley-Fenner, G., Carey, S., \& Solimando, A. (2002). Objects are individuals but stuff doesn't count: Perceived rigidity and cohesiveness influence infants' representations of small numbers of discrete entities. Cognition, 85, 203-221.

Izard, V., Dehaene-Lambertz, G., \& Dehaene, S. (2008). Distinct cerebral pathways for object identity and number in human infants. $P u b$ lic Library of Science- Biology, 6(2), e11. doi:10.1371/journal.pbio.0060011

Kiefer, M., \& Dehaene, S. (1997). The time course of parietal activation in single-digit multiplication: Evidence from event-related potentials. Mathematical Cognition, 3, 1-30. doi:10.1080/135467997387461
Kobayashi, T., Hiraki, K., Mugitani, R., \& Hasegawa, T. (2004). Baby arithmetic: One object plus one tone. Cognition, 91, B23-B34. doi:10.1016/j. cognition.2003.09.004

Koechlin, E., Dehaene, S., \& Mehler, J. (1997). Numerical transformations in five month old human infants. Mathematical Cognition, 2, 89104. doi:10.1080/135467997387425

Kuhl, P. K. (2004). Early language acquisition: Cracking the speech code. Nature Reviews Neuroscience, 5, 831-843. doi:10.1038/nrn1533

Lemer, C., Dehaene, S., Spelke, E., \& Cohen, L. (2003). Approximate quantities and exact number words: Dissociable systems. Neuropsychologia, 41(14), 1942-1958. doi:10.1016/S00283932(03)00123-4

Lent, R. (2001). Cem bilhões de neurônios: Conceitos fundamentais de neurociência. São Paulo, SP: Atheneu.

Lewandowsky, M., \& Stadelmann, E. (1908). Ueber einen bemerkenswerten Fall von Himblutung und uber Rechenstorungen bei Herderkrankurg des Gehirns. Journal für Psychologie und Neurologie, 11, 249-265.

Lima, L. O. (1999). Piaget: Sugestões aos educadores. Petrópolis, RJ: Vozes.

Lipton, J. S., \& Spelke, E. S. (2003). Origins of number sense: Large-number discrimination in human infants. Psychological Science, 14(5), 396401. doi:10.1111/1467-9280.01453

Lipton, J. S., \& Spelke, E. S. (2004). Discrimination of large and small numerosities by human infants. Infancy, 5(3), 271-290. doi:10.1207/ s15327078in0503 2

Mandler, G., \& Shebo, B. J. (1982). Subitizing: An analysis of its component processes. Jounal of Experimental Psychology, 111, 1-21. doi:10.1037/0096-3445.111.1.1

Nieder, A. (2005). Counting on neurons: The neurobiology of numerical competence. Nature Reviews Neuroscience, 6, 177-190. doi:10.1038/ nrn1626

Pica, P., Lemer, C., Izard, V., \& Dehaene, S. (2004). Exact and approximate arithmetic in an Amazonian indigene group. Science, 306, 499-503. doi:10.1126/science.1102085

Pinel, P., Dehaene, S., Riviere, D., \& LeBihan, D. (2001). Modulation of parietal activation by semantic distance in a number comparison 
task. Neuroimage, 14, 1013-1026. doi:10.1006/ nimg.2001.0913

Prado, P. S. T. (2010). Números e linguagem. In J. S. Carmo \& P. S. T. Prado (Eds.), Relações simbólicas e aprendizagem da matemática (pp. 273303). Santo André, SP: Esetec.

Simon, T., Hespos, S., \& Rochat, P. (1995). Do infants understand simple arithmetic? A replication of Wynn (1992). Cognitive Development, 10, 253-269. doi:10.1016/0885-2014(95) 90011-X

Spelke, E. S. (2003). What makes humans smart? Core knowledge and natural language. In D. Gentner \& S. Goldin-Meadow (Eds.), Language in mind (pp. 277-311) Cambridge, MA: Massachusetts Institute of Technology Press.

Starkey, P. (1992). The early development of numerical reasoning. Cognition, 43, 93-126. doi:10.1016/0010-0277(92)90034-F

Starkey, P., \& Cooper, R. G., Jr. (1980). Perception of numbers by human infants. Science 210 , 1033-1035.

Starkey, P., Spelke, E. S., \& Gelman, R. (1990). Numerical abstraction by human infants. Cognition, 36, 97-128

Temple, C. M. (1989). Digit dyslexia: A categoryspecific disorder in developmental dyscalculia. Cognitive Neuropsychology, 6, 93-116. doi:10.1080/02643298908253287

Trick, L., \& Pylyshyn, Z. W. (1994). Why are small and large numbers enumerated differently? A limited capacity preattentive stage in vision. Psychological Review, 101, 80-102. doi:10.1037/0033-295X.101.1.80
Van de Walle, G., Carey, S., \& Prevor, M. (2000). Bases for object individuation in infancy: Evidence from manual search. Journal of Cognition and Development, 1, 249-280.

Varley, R. A., Klessinger, N. J., Romanowski, C. A., \& Siegal, M. (2005). Agrammatic but numerate. Proceedings of the National Academy of Science, 102(9), 3519-3554. doi:10.1073/ pnas.0500328102

Wynn, K. (1992a). Addition and subtraction by human infants. Nature, 358, 749-750. doi: $10.1038 / 358749 \mathrm{a} 0$

Wynn, K. (1992b). Children's acquisition of the number words and the counting system. Cognitive Psychology, 24, 220-251. doi:10.1016/0010-0285(92)90008-P

Wynn, K. (1996). Infants' individuation and enumeration of actions. Psychological Science, 7, 164169. doi:10.1111/j.1467-9280.1996.tb00350.x

Wynn, K. (2002). Do infants have numerical expectations or just perceptual preferences? Developmental Science, 2, 207-209. doi:10.1111/14677687.002213

Xu, F., \& Spelke, E. S. (2000). Large number discrimination in 6-month old infants. Cognition, 74(1), B1-B11.

Xu, F., Spelke, E. S., \& Goddard, S. (2005). Number sense in human infants. Developmental Science, 8(1), 88-101. doi:10.1111/j.14677687.2005.00395.x 